hep-th/0201232

SINP-TNP/02-7

\title{
Correlators Of Open D-string Theory From Supergravity
}

\author{
Somdatta Bhattacharya and Shibaji Roy \\ Saha Institute of Nuclear Physics, 1/AF Bidhannagar, Calcutta-700 064, India \\ E-Mails: som,roy@theory.saha.ernet.in
}

\begin{abstract}
We consider a minimally coupled massless scalar propagating in the background of NS5branes in the presence of a 2 -form RR electric field. The supergravity solution also called the (NS5,D1) bound state in the appropriate decoupling limit is the holographic dual of Open Dstring (OD1) theory. Using this information, we compute the two-point function of the operators in OD1 theory which couples to the massless string states such as the dilaton. We will indicate how to obtain the absorption cross-section of a scalar on NS5-branes (i.e. Little String Theory (LST)) obtained earlier, from our results in the low-energy limit.
\end{abstract}

\section{Introduction}

It is well-known [1] from world-volume arguments that in six space-time dimensions there exist a series of new non-gravitational non-local theories which appear as particular decoupling limits of type IIB or IIA NS5-branes in the presence of $(p+1)$-form RR electric gauge fields. As the world-volume theory decouples from the bulk, the corresponding士 $(p+1)$-form field strength attains a critical value and almost balances the tension of the $\mathrm{D} p$-branes living on the world-volume of NS5-branes. Thus these theories contain excitations which include fluctuating light open $\mathrm{D} p$-branes and are called OD $p$ theories. The idea for the existence of such theories came from a series of S- and T-duality transformations of the underlying string theory starting from Non-Commutative Yang-Mills

\footnotetext{
${ }^{1}$ What we mean by 'corresponding' here is that the $(p+1)$-form gauge field in the supergravity solution becomes the $(p+1)$-form field-strength on the world-volume by gauge invariance.
} 
(NCYM) theory [2, 3, 4, 5] in 3+1 dimensions. To be precise NCYM appears as a generalization of the AdS/CFT correspondence [6, 7] on the world volume of D3-branes in the presence of a constant NSNS $B$ field with space-space components [2]. By S-duality it has been argued that the strong coupling limit of NCYM theory does not give rise to a field theory, rather it gives a non-local theory called a Non-Commutative Open String (NCOS) theory in $3+1$ dimensions [8, 9]. By applying T-duality on this $3+1$ dimensional NCOS theory one obtains other NCOS theories in various dimensions [10], where the 2-form field strength corresponding to the NSNS 2-form gauge field attains a critical value. S-duality on the six-dimensional NCOS theory then gives rise to six-dimensional OD1 theory, where now the 2-form field strength corresponding to the RR 2-form gauge field takes the critical value [1, 11]. By applying T-duality again on the OD1-theory one obtains the series of six-dimensional theories called the ODp-theories [1] with coupling constant $G_{o(p)}^{2}$ and length scale $\sqrt{\alpha_{\text {eff }}^{\prime}}$. In this paper, we will mainly be interested in OD1 theory, with coupling $G_{o(1)}^{2}$ and length scale $\sqrt{\alpha_{\text {eff }}^{\prime}}$.

Holographic dual descriptions of these various world volume theories are given by the corresponding bound state solutions (having sixteen super charges) of the supergravity equations of motion with appropriate decoupling limits. Thus the holographic dual of $3+1$ NCYM theory is the (D1,D3) bound state [12, 13, 14] with the NCYM decoupling limit [2, 3, 田. Similarly $(\mathrm{F}, \mathrm{D} p)$ bound states 15 with NCOS decoupling limits are the holographic duals of NCOS theories in $(p+1)$ dimensions [10]. The holographic duals of $\mathrm{OD} p$ theories in six dimensions are given by the (NS5,D $p)$ bound state solutions [11, 16, 17, 18] with OD $p$ decoupling limits. Since we will be working with OD1 theory, we will consider only the (NS5,D1) bound state solution of type IIB string theory.

Since OD $p$ theories are defined on the world-volume of NS5 branes and it is not known how to quantize 5-branes, very little is known about the dynamics of such theories. So for example, we only know that OD1 theory has a coupling $G_{o(1)}^{2}$ and length scale $\sqrt{\alpha_{\text {eff }}^{\prime}}$ and it must reduce to Little String Theory [19, 20, 21, 22, 23] at low energies where $G_{o(1)}^{2} \rightarrow 0$ and $\alpha_{\text {eff }}^{\prime}=$ fixed. So in the absence of our knowledge about the world volume dynamics, we will use its holographic description and try to gain some insight into this theory. The (NS5,D1) bound state solution and the associated OD1 decoupling limit have been obtained in [1, 16, 17]. We study the propagation of a massless scalar minimally coupled to this background geometry. We note that the background geometry in the string frame becomes asymptotically flat, whereas in the near horizon limit it is given by the (NS5,D1) supergravity metric with the OD1 limit. Unlike in the D3 brane case, the dilaton is not a constant here and so we cannot trust the (NS5,D1) supergravity solution all the way through the energy $u \rightarrow 0$ in the boundary theory and we have to use the S-dual solution 
i.e. the (F,D5) supergravity solution [15] in the corresponding NCOS limit [10]. However, the equation of motion and therefore the solution remains the same in terms of the dual variables. After finding the unique solution to the minimally coupled scalar, $\varphi(k, u)$, we extract the two-point function of the operator $\mathcal{O}(k)$ in the boundary OD1 theory dual to the field $\varphi$ by the usual procedure [24, 25]. To be exact, we identify in the spirit of the AdS/CFT correspondence

$$
\left\langle e^{i \int d^{6} k \varphi_{0}(k) \mathcal{O}(k)}\right\rangle=e^{i S_{\text {sugra }}\left[\left.\varphi(k, u)\right|_{u=\Lambda}\right]}
$$

where on the r.h.s. $S_{\text {sugra }}$ represents the on-shell supergravity action of the scalar $\varphi(k, u)$ in the background geometry mentioned above. That is on imposing equation of motion, the scalar action gives a boundary term which has to be evaluated at the boundary $u=\Lambda$, where $\left.\varphi(k, u)\right|_{u=\Lambda} \rightarrow \varphi_{0}(k)$, with proper renormalization. Since the l.h.s. represents the generating functional of the operator $\mathcal{O}(k)$ of the boundary theory, the two point function can be evaluated from $S_{\text {sugra }}$ by taking the functional derivative with respect to $\varphi_{0}(k)$ twice. We will evaluate the correlation function only in momentum space as the operators are always well defined in momentum space for a translationally invariant theory. But as we will see, because of the momentum dependent renormalization, it is not possible to obtain the correlator in position space unambiguously [26, [4 which is indicative of the non-local nature of the boundary theory. We will also point out how in the low energy limit, our result reproduces the form of the absorption cross-section of a scalar on NS5-branes [27, 26] or Little String Theory (LST) as expected.

The organization of this paper is as follows. In section 2, we give the holographic dual description of OD1 theory. The computations of the boundary action and the two-point function of operators in OD1 theory are given in section 3. Here we also mention how at low energy we reproduce the absorption cross-section of LST. In section 4 we present our conclusion.

\section{Holographic dual of OD1 theory}

As we mentioned in the introduction, the holographic dual description of OD1 theory is given by the (NS5,D1) supergravity solution in a particular low energy limit called the OD1 decoupling limit. The string-metric and the dilaton for the above bound state are given as follows $[17]^{2}$,

$$
d s^{2}=H^{\frac{1}{2}} H^{\prime \frac{1}{2}}\left[H^{-1}\left(-\left(d x^{0}\right)^{2}+\left(d x^{1}\right)^{2}\right)+H^{\prime-1} \sum_{i=2}^{5}\left(d x^{i}\right)^{2}+d r^{2}+r^{2} d \Omega_{3}^{2}\right]
$$

\footnotetext{
${ }^{2}$ For our purpose here we do not need the other fields which can be found in 17 .
} 


$$
e^{\phi}=g_{s} H^{\frac{1}{2}}
$$

In the above ' $r$ ' is the radial coordinate transverse to the NS5-branes. The D-strings lie along the $x^{1}$ direction. Also, $d \Omega_{3}^{2}=d \theta^{2}+\sin ^{2} \theta d \phi_{1}^{2}+\sin ^{2} \theta \sin ^{2} \phi_{1} d \phi_{2}^{2}$ is the line element of the unit sphere transverse to NS5-branes. $g_{s}=e^{\phi_{0}}$ is the string coupling constant. The harmonic functions have the forms

$$
\begin{aligned}
H & =1+\frac{Q_{5}}{r^{2}} \\
H^{\prime} & =1+\frac{\cos ^{2} \psi Q_{5}}{r^{2}}
\end{aligned}
$$

where the charge $Q_{5}$ and the angle $\cos \psi$ are given as follows,

$$
\begin{aligned}
Q_{5} & =\frac{N \alpha^{\prime}}{\cos \psi} \\
\cos \psi & =\frac{N}{\sqrt{M^{2} g_{s}^{2}+N^{2}}}
\end{aligned}
$$

with $N$, the number of NS5-branes and $M$, the number of D-strings per $(2 \pi)^{4} \alpha^{2}$ of four co-dimensional area of NS5-branes. The OD1 decoupling limit [1, 16, 17] is obtained by defining a dimensionless scaling parameter $\epsilon$ and taking $\cos \psi=\epsilon \rightarrow 0$, keeping the following quantities fixed,

$$
\alpha_{\mathrm{eff}}^{\prime}=\frac{\alpha^{\prime}}{\epsilon}, \quad u=\frac{r}{\epsilon \alpha_{\mathrm{eff}}^{\prime}}, \quad G_{o(1)}^{2}=\frac{g_{s}}{\epsilon}, \quad Q_{5}=\alpha_{\mathrm{eff}}^{\prime} N
$$

With these the harmonic functions in (2.2) take the forms,

$$
\begin{aligned}
H & =\frac{1}{\epsilon^{2} a^{2} u^{2}} \\
H^{\prime} & =\frac{h}{a^{2} u^{2}}
\end{aligned}
$$

where $h=1+a^{2} u^{2}$, with $a^{2}=\alpha_{\text {eff }}^{\prime} / N$ and the metric and the dilaton reduce to

$$
\begin{aligned}
d s^{2} & =\alpha^{\prime} h^{\frac{1}{2}}\left[-\left(d \tilde{x}^{0}\right)^{2}+\left(d \tilde{x}^{1}\right)^{2}+h^{-1} \sum_{i=2}^{5}\left(d \tilde{x}^{i}\right)^{2}+\frac{N}{u^{2}}\left(d u^{2}+u^{2} d \Omega_{3}^{2}\right)\right] \\
e^{\phi} & =\frac{G_{o(1)}^{2}}{a u}
\end{aligned}
$$

In the above $\sqrt{\alpha_{\text {eff }}^{\prime}}$ is the length scale, $u$ is the energy parameter and $G_{o(1)}^{2}$ is the coupling constant of OD1 theory. Note that even though $u$ is fixed, it can be set to an arbitrarily 
small or large values corresponding to the IR or UV limit of OD1 theory. The fixed coordinates are defined as

$$
\tilde{x}^{0,1}=\frac{1}{\sqrt{\alpha_{\text {eff }}^{\prime}}} x^{0,1}, \quad \tilde{x}^{2, \ldots, 5}=\frac{1}{\epsilon \sqrt{\alpha_{\text {eff }}^{\prime}}} x^{2, \ldots, 5}
$$

Eq.(2.6) represents the supergravity dual of OD1-theory.

The supergravity description remains valid as long as the curvature measured in units of $\alpha^{\prime}$ i.e. $\alpha^{\prime} \mathcal{R}=1 /\left(1+a^{2} u^{2}\right)^{\frac{1}{2}} N \ll 1$ and the dilaton $e^{\phi}=G_{o(1)}^{2} /(a u) \ll 1$. This can be satisfied if $N$ is large and $a u \gg G_{o(1)}^{2}$. So in the extreme IR region $(u \rightarrow 0)$, we cannot trust the supergravity solution (2.6) and we have to use its S-dual version i.e. the (F,D5) bound state with the corresponding NCOS decoupling limit [28]. But we will point out later that this will not change the results.

We would like to mention that in the low-energy limit we would expect that the OD1 theory would reduce to LST [1]. However, this is not quite transparent from the OD1 limit in (2.4) and the supergravity configuration (2.6). In fact, the OD1-limit looks quite different from the LST limit $g_{s} \rightarrow 0$ and $\alpha^{\prime}=$ fixed on NS5-branes as has been also pointed out in refs. 16, 17]. The reason can be understood from the angle cos $\psi$ given in (2.3). Notice here that since $\cos \psi=\epsilon \rightarrow 0$, the number of D-strings is very large compared to the number of NS5-branes. Since D-strings dominate over NS5-branes, it is not clear how to obtain the NS5-brane world-volume theory only, in the OD1 limit. Even from the metric in $(2.6)$, although in the low-energy limit $h \approx 1$, and we recover six dimensional Poincare invariant theory, it does not look like the holographic dual of LST, because the metric is multiplied with $\alpha^{\prime} \rightarrow 0$ (instead of some fixed $\alpha^{\prime}$ ). A decoupling limit different from (2.4) which closely resembles the LST limit has been discussed in [11]. This limit has been referred to as the OBLST limit and from here it is easy to recover the holographic dual of LST in the low-energy limit. But this type of limit holds only for (NS5,D1) and $(\mathrm{NS} 5, \mathrm{D} 2)$ and cannot be generalised for other $(\mathrm{NS} 5, \mathrm{D} p)$ cases. This is the reason we work with the OD1-limit (2.4).

The clue to recover LST from (2.6) is the fact that we have to take $G_{o(1)}^{2} \rightarrow 0$ with $\alpha_{\text {eff }}^{\prime}$ fixed. To see precisely how this can be achieved one should look closely at the angle given in (2.3). Notice that since $\cos \psi=\epsilon \rightarrow 0$, it implies that $N / M g_{s}=N /\left(M G_{o(1)}^{2} \epsilon\right) \sim \epsilon$. So if $G_{o(1)}^{2}=$ fixed, then $N / M \sim \epsilon^{2}$; on the other hand, if $G_{o(1)}^{2} \sim \epsilon \rightarrow 0$, such that $\cos \psi \approx 1$, then both $H$ and $H^{\prime}$ in (2.2) are the same and have the values $H=H^{\prime}=1 /\left(a^{2} \epsilon^{2} u^{2}\right)$. Using this and $u=r /\left(\epsilon \alpha_{\text {eff }}^{\prime}\right)$, it can be easily checked that (2.1) indeed reduces to the holographic dual of LST [29]. Note that in this case $\alpha^{\prime}=\alpha_{\text {eff }}^{\prime}=$ fixed. The parameter $\epsilon$ is then defined by the relation $u=r /\left(\epsilon \alpha_{\text {eff }}^{\prime}\right)$. Thus we conclude that LST is the low-energy 
limit of OD1 theory. Since $(5+1)$ dimensional NCOS theory is S-dual to OD1 theory, the coupling constant and the length scale $G_{o}^{2}$ and $\sqrt{\tilde{\alpha}_{\text {eff }}^{\prime}}$ of NCOS theory are related to those of OD1 theory by $G_{o}^{2}=1 / G_{o(1)}^{2}$ and $\sqrt{\tilde{\alpha}_{\text {eff }}^{\prime}}=G_{o(1)} \sqrt{\alpha_{\text {eff }}^{\prime}}$. So in order to recover LST from NCOS theory we should take $G_{o}^{2} \rightarrow \infty$ and $\tilde{\alpha}_{\text {eff }}^{\prime} \rightarrow 0$, such that $G_{o}^{2} \tilde{\alpha}_{\text {eff }}^{\prime}=\alpha_{\text {eff }}^{\prime}=$ fixed. One can check by a similar argument that LST can indeed be obtained in this limit.

In the next section when we compute the correlator of OD1 theory we will point out how to obtain the absorption cross-section of a minimally coupled massless scalar on NS5-branes or LST obtained before [26, 27, from our result in the low energy limit.

\section{Boundary action and the two point function of OD1 theory}

Here we consider the propagation of a minimally coupled massless scalar $\varphi$ in the background geometry of the (NS5,D1) bound state solution given in eq.(2.1) in the corresponding decoupling limit. The scalar $\varphi$ corresponds to the fluctuation of the dilaton (or it could be certain longitudinal components of the graviton). Setting all other fluctuations to zero, the action for the scalar can be written as,

$$
S_{\text {sugra }}=\frac{1}{(2 \pi)^{7} \alpha^{\prime 4}} \int d^{10} x \sqrt{-g} e^{-2 \phi}\left[\frac{1}{2} g^{\mu \nu} \partial_{\mu} \varphi \partial_{\nu} \varphi\right]
$$

We have written the action in the string frame. Using the background geometry given in (2.1) and assuming that $\varphi$ is in the $s$-wave, the above action can be written as follows,

$$
S_{\text {sugra }}=\frac{1}{4(2 \pi)^{5} g_{s}^{2} \alpha^{4}} \int d^{6} x \int d r r^{3}\left[\left(\partial_{r} \varphi\right)^{2}+H \eta^{\mu \nu} \partial_{\mu} \varphi \partial_{\nu} \varphi+H^{\prime} \delta^{i j} \partial_{i} \varphi \partial_{j} \varphi\right]
$$

where in (3.2) we have performed the integration over the angular part yielding a factor $\Omega_{3}=2 \pi^{2}$. The indices $\mu, \nu=0,1$ and $i, j=2, \ldots, 5 . H$ and $H^{\prime}$ are harmonic functions given in (2.2). The equation of motion following from (3.2) is

$$
\left[\frac{1}{r^{3}} \partial_{r}\left(r^{3} \partial_{r}\right)+H \eta^{\mu \nu} \partial_{\mu} \partial_{\nu}+H^{\prime} \delta^{i j} \partial_{i} \partial_{j}\right] \varphi=0
$$

In the OD1-decoupling limit (2.4), the above equation reduces to

$$
\left[\frac{1}{u^{3}} \partial_{u}\left(u^{3} \partial_{u}\right)-\frac{N k^{2}}{u^{2}}-k^{\prime 2}\right] \tilde{\varphi}(u)=0
$$

where,

$$
\varphi\left(x^{\mu}, r\right)=e^{i k \cdot x} \tilde{\varphi}(u) \varphi_{0}(k)
$$


with $\mu=0,1, \ldots, 5$ and $k \cdot x=\sum_{\mu=0}^{5} k_{\mu} x^{\mu}$. Also in (3.4) we have defined $k^{2}=\sum_{i=1}^{5} \tilde{k}_{i}^{2}-\tilde{\omega}^{2}$ and $k^{\prime 2}=\alpha_{\text {eff }}^{\prime} \sum_{j=2}^{5} \tilde{k}_{j}^{2}$.

The 'tilde' variables are the scaled variables and are related to the original variables as $\tilde{\omega}=\sqrt{\alpha_{\text {eff }}^{\prime}} \omega, \tilde{k}_{1}=\sqrt{\alpha_{\text {eff }}^{\prime}} k_{1}$ and $\tilde{k}_{j}=\epsilon \sqrt{\alpha_{\text {eff }}^{\prime}} k_{j}$, for $j=2, \ldots, 5$. After defining the scaled variables this way, we will drop the 'tildes' for notational simplicity from now on. We recognize eq.(3.4) to be the equation for the modified Bessel function with two independent solutions given by

$$
\tilde{\varphi}(u)=u^{-1} I_{\nu}\left(k^{\prime} u\right) \quad \text { or } \quad u^{-1} K_{\nu}\left(k^{\prime} u\right)
$$

where $k^{\prime}=\sqrt{k^{\prime 2}}$ and $\nu^{2}=1+N k^{2}$, with $I_{\nu}$ and $K_{\nu}$ being the modified Bessel functions. From these the solution which will be chosen must be well behaved as $u \rightarrow 0$. So, the unique solution that is picked would be,

$$
\tilde{\varphi}(u)=u^{-1} I_{\nu}\left(k^{\prime} u\right)
$$

A word of caution is needed at this point. We have mentioned earlier that the (NS5,D1) supergravity solution may not be trustworthy at arbitrarily small values of the energy parameter, since it may violate the condition $e^{\phi}=G_{o(1)}^{2} /(a u) \ll 1$. In that case one has to use the S-dual configuration i.e. (F,D5) solution with the corresponding NCOS limit. But one can check that this will lead to the same equation of motion giving the same solution as in the (NS5,D1) case. Now, using the following identity,

$$
I_{\nu}\left(k^{\prime} u\right)=e^{-i \nu \pi / 2} J_{\nu}\left(i k^{\prime} u\right)
$$

where $J_{\nu}$ is the ordinary Bessel function, we obtain the following asymptotic behaviour of $\tilde{\varphi}(u)$,

$$
\tilde{\varphi}(u) \rightarrow \frac{1}{\sqrt{2 \pi k^{\prime}} u^{\frac{3}{2}}}\left[e^{-k^{\prime} u} e^{-i\left(\nu+\frac{1}{2}\right) \pi}+e^{k^{\prime} u}\right]
$$

for $u \rightarrow \infty$. Note here that the coefficient of $e^{-k^{\prime} u}$ in (3.9) is like the reflection coefficient for the scalar. However, we would like to point out that since $k^{\prime}$ does not include the time-like momenta, so this is not really the reflection coefficient. If on the other hand, we had set all the space-like momenta to zero, then the equation of motion (3.4) would reduce to

$$
\left[\frac{1}{u^{3}} \partial_{u}\left(u^{3} \partial_{u}\right)+\frac{N \tilde{\omega}^{2}}{u^{2}}\right] \tilde{\varphi}(u)=0
$$

The solution for this equation has the form

$$
\tilde{\varphi}(u)=u^{-1 \pm \sqrt{1-N \tilde{\omega}^{2}}}
$$


So in this case there will be non-trivial absorption if

$$
\tilde{\omega}>\frac{1}{\sqrt{N}} \quad \text { or } \quad \omega>\frac{1}{\sqrt{\alpha_{\text {eff }}^{\prime} N}}
$$

This is similar to the mass gap found for LST [26, 30].

Now in order to evaluate the two-point function, it has to be properly renormalised. We here adopt the technique used in [26, 团 for this purpose. Defining $u=\Lambda$ (where $\Lambda$ is the UV cutoff of the OD1 theory) as the boundary, we demand the following boundary condition for our solution $\tilde{\varphi}(u)$,

$$
\tilde{\varphi}(\Lambda)=\frac{1}{\sqrt{2 \pi k^{\prime}}} \frac{1}{\Lambda^{\frac{3}{2}}} e^{k^{\prime} \Lambda} \quad \text { as } \quad \Lambda \rightarrow \infty
$$

This condition ensures that the solution remains finite in the interior as $\Lambda \rightarrow \infty$. We are now in a position to evaluate the boundary action. Integrating by parts and using (3.3), we obtain from $(3.2)$

$$
\begin{aligned}
S_{\text {sugra }} & =\frac{1}{2(2 \pi)^{5} g_{s}^{2} \alpha^{44}} \int d^{6} x \int d r \partial_{r}\left(r^{3} \varphi \partial_{r} \varphi\right) \\
& =\frac{\alpha_{\text {eff }}^{\prime}}{2(2 \pi)^{5} G_{o(1)}^{4}} \int d^{6} x \int d u \partial_{u}\left(u^{3} \varphi \partial_{u} \varphi\right)
\end{aligned}
$$

Expanding $\varphi\left(x^{\mu}\right)$ as

$$
\varphi\left(x^{\mu}\right)=\int d^{6} k e^{i k \cdot x} \varphi_{0}(k)
$$

eq.(3.14) can be simplified to give

$$
S_{\text {sugra }}=\frac{\pi \alpha_{\text {eff }}^{\prime}}{G_{o(1)}^{4}} \int d^{6} k d^{6} q \delta^{6}(k+q) \varphi_{0}(k) \varphi_{0}(q) \mathcal{F}
$$

The flux factor $\mathcal{F}$ is defined as

$$
\left.\mathcal{F}=\tilde{\varphi}(u) u^{3} \partial_{u} \tilde{\varphi}(u)\right]_{u=0}^{u=\Lambda}
$$

Since $\varphi$ falls off exponentially as $u \rightarrow 0$, we need to evaluate (3.17) for $u=\Lambda$ (cutoff surface). Using (3.9) and (3.13) in (3.16), we obtain the supergravity action on the boundary as,

$$
S_{\text {sugra }}=\frac{\pi \alpha_{\text {eff }}^{\prime}}{G_{o(1)}^{4}} \int d^{6} k\left[\operatorname{div}(\Lambda, k)-\frac{1}{2 \pi} e^{-i\left(\nu+\frac{1}{2}\right) \pi}+\ldots . .\right] \varphi_{0}(k) \varphi_{0}(-k)
$$

The first term is divergent as $\Lambda \rightarrow \infty$ and should be subtracted away. By the 'dots' we indicate terms that vanish as $\Lambda \rightarrow \infty$. So the two point function has the form

$$
\langle\mathcal{O}(k) \mathcal{O}(-p)\rangle \sim \frac{\alpha_{\text {eff }}^{\prime}}{2 G_{o(1)}^{4}} e^{-i \nu \pi} \delta^{6}(k-p)
$$


It is clear that if $\nu$ is real i.e. if $1+N\left(\sum_{i=1}^{5} k_{i}^{2}-\omega^{2}\right)>0$, then the correlation function is trivial in the sense that it becomes an analytic function of the momentum. On the other hand if we define $\left[1+N\left(\sum_{i=1}^{5} k_{i}^{2}-\omega^{2}\right)\right]^{1 / 2}=-i \mu$, for $\mu=$ real, then the correlation function is non-trivial and has the form,

$$
\langle\mathcal{O}(k) \mathcal{O}(-p)\rangle \sim \frac{\alpha_{\mathrm{eff}}^{\prime}}{2 G_{o(1)}^{4}} e^{-\mu \pi} \delta^{6}(k-p)
$$

This is our result for the two-point function of an operator in the OD1 theory which couples to the massless scalar in supergravity. For small values of $\mu$, eq.(3.20) reduces to

$$
\langle\mathcal{O}(k) \mathcal{O}(-p)\rangle \sim \frac{\alpha_{\text {eff }}^{\prime} \pi}{2 G_{o(1)}^{4}}\left[N\left(\omega^{2}-\sum_{i=1}^{5} k_{i}^{2}\right)-1\right]^{1 / 2} \delta^{6}(k-p)
$$

Note that even if there is no real absorption here (see eq.(3.9) and the remarks thereafter), we still get a non-trivial correlation function.

Now we will show how the absorption cross-section of a scalar on the NS5-brane theory obtained before [27, 26] can be deduced in the low-momentum limit. Note that for low momentum we can rewrite the equation of motion (3.4) as,

$$
\left[\frac{1}{u^{3}} \partial_{u}\left(u^{3} \partial_{u}\right)-\frac{N k^{2}}{u^{2}}-k^{2}\right] \tilde{\varphi}(u)=0
$$

The solution for this equation would be given as

$$
\tilde{\varphi}(u)=u^{-1} I_{\nu}(k u)
$$

The asymptotic behaviour of this function is

$$
\tilde{\varphi}(u) \rightarrow \frac{1}{\sqrt{2 \pi k} u^{\frac{3}{2}}}\left[e^{-k u} e^{-i\left(\nu+\frac{1}{2}\right) \pi}+e^{k u}\right]
$$

Unlike in the case (3.9), $k$ now includes the time-like momentum and the coefficient of $e^{-k u}$ is truly the reflection coefficient of a scalar. So if $\nu$ is real then $|R|^{2}=1$ and there is no absorption. In the absence of longitudinal momenta, the reflection coefficient would be given as

$$
|R|^{2}=e^{-2 \pi \mu}
$$

where $\mu=\sqrt{N \alpha^{\prime} \omega^{2}-1}$.

Therefore, the absorption cross-section would be proportional to

$$
\sigma \sim\left(1-e^{-2 \pi \mu}\right)
$$

\footnotetext{
${ }^{3}$ For large $u$, we should not neglect 1 in both the harmonic functions $H$ and $H^{\prime}$ in (2.2). Then it can be checked from (3.3) that the equation of motion has the form (3.22) even for large $u$.
} 
This result was obtained in refs. [27, 26]. We have thus recovered the absorption crosssection of a scalar on the NS5-brane or LST in the low-energy limit. Note that since it is the coefficient of $e^{-k u}$ which determines the form of the correlation function and they have the same exponential form in both OD1 theory and NS5 brane theory, the correlation function will have the same structure in this case as in (3.20). But of course $\mu$ is different in this case and is given by $\mu=\left[N \alpha^{\prime}\left(\omega^{2}-\sum_{i=1}^{5} k_{i}^{2}\right)-1\right]^{1 / 2}$ with $\alpha^{\prime}$ fixed, whereas for OD1 theory $\mu=\left[N\left(\tilde{\omega}^{2}-\sum_{i=1}^{5} \tilde{k}_{i}^{2}\right)-1\right]^{1 / 2}$, where the scaled variables were defined before.

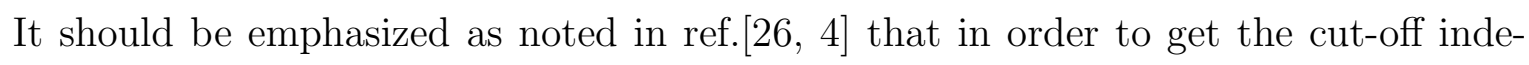
pendent correlation function we had to perform a momentum dependent renormalization. Because of this, it is not possible to Fourier transform the momentum-space correlation function to position space in an unambiguous manner. This is related to the non-local nature of the boundary theory in the UV.

It is not difficult to generalize our result for the higher partial waves. The $s$-wave equation of motion (3.3), should be modified for the $l$-th partial waves as,

$$
\left[\frac{1}{r^{3}} \partial_{r}\left(r^{3} \partial_{r}\right)+H \eta^{\mu \nu} \partial_{\mu} \partial_{\nu}+H^{\prime} \delta^{i j} \partial_{i} \partial_{j}-\frac{l(l+2)}{r^{2}}\right] \varphi=0
$$

In the OD1-decoupling limit (2.4), it reduces to

$$
\left[\frac{1}{u^{3}} \partial_{u}\left(u^{3} \partial_{u}\right)-\frac{N k^{2}}{u^{2}}-k^{\prime 2}-\frac{l(l+2)}{u^{2}}\right] \tilde{\varphi}(u)=0
$$

Following the same technique as before we obtain the form of the two-point function as

$$
\langle\mathcal{O}(k) \mathcal{O}(-p)\rangle \sim \frac{\alpha_{\text {eff }}^{\prime}}{2 G_{o(1)}^{4}} e^{-\mu \pi} \delta^{6}(k-p)
$$

where now,

$$
\mu=\left[N\left(\omega^{2}-\sum_{i=1}^{5} k_{i}^{2}\right)-(l+1)^{2}\right]^{\frac{1}{2}}
$$

Note here that $\omega$ and $k_{i}$ 's are scaled variables as in eq.(3.21). This concludes our calculation of two point function for the operators in OD1 theory which couple to the massless string states.

\section{Conclusion}

With very little knowledge at hand about the world-volume theory of NS5-brane in the presence of a near critical RR 2-form electric field, or OD1 theory, we have taken recourse to its holographic description to gain some insight into this theory. The (NS5,D1) bound 
state solution of type IIB supergravity in a particular low energy limit called the OD1 decoupling limit is the holographic description of OD1 theory. We have studied the propagation of a massless scalar minimally coupled to this background. We computed the boundary action and in the spirit of AdS/CFT correspondence we obtained the form of two point function of the operators in OD1 theory which couples to massless string states such as the dilaton. In order to get a finite correlation function, we had to perform a momentum dependent renormalization for the scalar $\varphi$ and this is the reason we can not Fourier transform the momentum space correlation function into position space in an unambiguous way. This is a reflection of the fact that the boundary theory is non-local. We have also pointed out how to obtain the absorption cross-section of a scalar on NS5branes from our results in the low energy limit. Finally, we have extended our results for the higher partial waves.

In the case of coincident D3-branes, coupling this background to massive string states one can compute the correlation function of the operators in the Yang-Mills theory which couple to massive string modes. The position space correlation function then determines the anomalous dimensions of the operators in Yang-Mills theory [24, 25]. For the case of (NS5,D1) background also, it is possible to couple it to massive string states. However, we have not been able to solve the corresponding equation of motion. In any case, since the boundary OD1 theory is non-local, it will not be possible to obtain the position space correlation function in an unambiguous way and hence the determination of the dimension of such an operator would also be ambiguous.

We have not studied in this paper the structure of the correlation function for other $\mathrm{OD} p$ theories. For odd $p$, the corresponding holographic description would be given by the type IIB supergravity background in the appropriate decoupling limits. So, the correlation function in this case will have similar structure as found in this paper. However, for $p$ $=$ even, the holographic description would be given by type IIA supergravity background and for $u \rightarrow 0$, the appropriate description would be given by uplifting the solution to Mtheory. It would be interesting to understand the structure of correlation function in this case which in the low energy limit should reproduce the form of the correlation function of LST obtained in ref. [26]. It would also be interesting to understand the results of this paper from the world volume point of view along the line [31, 32].

\section{References}

[1] R. Gopakumar, S. Minwalla, N. Seiberg and A. Strominger, "(OM) theory in diverse dimensions", JHEP 08 (2000) 008, hep-th/0006062. 
[2] N. Seiberg, E. Witten, "String theory and noncommutative geometry", JHEP 09 (1999) 032, hep-th/9908142.

[3] A. Hashimoto and N. Itzhaki, "Noncommutative Yang-Mills and the AdS/CFT correspondence", Phys. Lett. B465 (1999) 142, [hep-th/9907166].

[4] J. Maldacena and J. Russo, "Large N limit of noncommutative gauge theories", JHEP 09 (1999) 025, hep-th/9908134.

[5] M. Alishahiha, Y. Oz and M. Sheikh-Jabbari, "Supergravity and large N noncommutative field theories", JHEP 11 (1999) 007, hep-th/9909215.

[6] J. Maldacena, "The large N limit of superconformal field theories and supergravity", Adv. Theor. Math. Phys. 2 (1998) 231, hep-th/9711200.

[7] O. Aharony, S. Gubser, J. Maldacena, H. Ooguri and Y. Oz, "Large N field theories, string theory and gravity", Phys. Rep. 323 (2000) 183, hep-th/9905111.

[8] N. Seiberg, L. Susskind and N. Toumbas, "Strings in background electric field space/time noncommutativity and a new noncritical string theory", JHEP 06 (2000) 021, hep-th/0005040.

[9] R. Gopakumar, J. Maldacena, S. Minwalla and A. Strominger, "S-duality and noncommutative gauge theory", JHEP 06 (2000) 036, hep-th/0005048.

[10] T. Harmark, "Supergravity and space-time noncommutative open string theory", JHEP 07 (2000) 043, hep-th/0006023.

[11] T. Harmark, "Open Branes in space-time noncommutative little string theory", Nucl. Phys. B593 (2001) 76, hep-th/0007147.

[12] J. Breckenridge, G. Michaud and R. Myers, "More D-brane bound states", Phys. Rev. D55 (1997) 6438, hep-th/9611174.

[13] J. Russo and A. Tseytlin, "Waves, boosted branes and BPS states in M-theory", Nucl. Phys. B490 (1997) 121, hep-th/9611047.

[14] M. Costa and G. Papadopoulos, "Superstring dualities and p-brane bound states", Nucl. Phys. B510 (1998) 217, hep-th/9611047.

[15] J. X. Lu and S. Roy, "Non-threshold (F, Dp) bound states", Nucl. Phys. B560 (1999) 181, hep-th/9904129. 
[16] M. Alishahiha, Y. Oz and J. Russo, "Supergravity and light-like noncommutativity", JHEP 09 (2000) 002, hep-th/0007215.

[17] I. Mitra and S. Roy, "(NS5, D $p)$ and (NS5, D $(p+2), \mathrm{D} p)$ bound states of type IIB and type IIA string theories", JHEP 02 (2001) 026, hep-th/0011236.

[18] M. Cederwall, U. Gran, M. Nielsen and B. E. W. Nilsson, " $(p, q)$ 5-branes in non-zero $B$ field", JHEP 01 (2000) 037, hep-th/9912106.

[19] M. Berkooz, M. Rozali and N. Seiberg, "Matrix description of M-theory on $T^{4}$ and $\mathrm{T}^{5}$ ", Phys. Lett. B408 (1997) 105, hep-th/9704089.

[20] N. Seiberg, "New theories in six dimensions and matrix description of M-theory on $\mathrm{T}^{5}$ and $\mathrm{T}^{5} / \mathrm{Z}_{2} "$, Phys. Lett. B408 (1997) 98, hep-th/9705221.

[21] R. Dijkgraaf, E. Verlinde and H. Verlinde, "BPS quantization of five-brane", Nucl. Phys. B486 (1997) 89, hep-th/9604055.

[22] R. Dijkgraaf, E. Verlinde and H. Verlinde, "5D black holes and matrix strings", Nucl. Phys. B506 (1997) 121, hep-th/9704018].

[23] A. Losev, G. Moore and S. Shatashvili, "M \& m's", Nucl. Phys. B522 (1998) 105, hep-th/9707250.

[24] S. Gubser, I. Klebanov and A. Polyakov, "Gauge theory correlators from non-critical string theory", Phys. Lett. B428 (1998) 105, hep-th/9802109.

[25] E. Witten, "Anti de Sitter space and holography", Adv. Theor. Math. Phys. 2 (1998) 253, hep-th/9802150.

[26] S. Minwalla and N. Seiberg, "Comments on the IIA NS fivebrane", JHEP 06 (1999) 007, hep-th/9903142.

[27] S. Gubser and I. Klebanov, "Absorption by branes and Schwinger terms in the world volume theory", Phys. Lett. B413 (1997) 41, hep-th/9708005.

[28] R. -G. Cai, I. Mitra, N. Ohta and S. Roy, "On decoupled theories in (5+1) dimensions from (F, D1, NS5, D5) supergravity configuration", JHEP 12 (2001) 029, hep-th/0111027. 
[29] N. Itzhaki, J. Maldacena, J. Sonnenschein and S. Yankielowicz, "Supergravity and large N limit of theories with sixteen supercharges", Phys. Rev. D58 (1998) 046004, [hep-th/9802042].

[30] M. Alishahiha, "On type II NS5 branes in the presence of an RR field", Phys. Lett. B486 (2000) 194, hep-th/0002198.

[31] O. Aharony, M. Berkooz, D. Kutasov and N. Seiberg, "Linear dilaton, NS5-branes and holography", JHEP 10 (1998) 004, hep-th/9808149.

[32] S. Elitzur, A. Giveon, D. Kutasov, E. Rabinovici and G. Sarkissian, "D-branes in the background of NS fivebranes", JHEP 08 (2000) 046, hep-th/0005052. 